\title{
Abdominal Wall Transplantation
}

\author{
Yash J. Avashia $^{1}$ - Gina A. Mackert ${ }^{1} \cdot$ Brian May $^{2} \cdot$ Detlev Erdmann $^{3}$. \\ Kadiyala V. Ravindra ${ }^{4}$
}

Published online: 1 July 2015

(C) Springer International Publishing AG 2015

\begin{abstract}
Abdominal wall vascularized composite allotransplantation (AW-VCA) is a relatively young field within transplantation with 21 reported cases to date in the last decade. All AW-VCAs have been performed on patients undergoing either simultaneous multivisceral transplantation (MVT) or intestinal transplantation (IT). The two approaches to AW-VCA described are macrovascular and microvascular approaches. While at the time that this review is written, only three institutions have performed this procedure; their outcomes have shown low allograft loss. In addition, acute rejection of the
\end{abstract}

This article is part of the Topical Collection on Vascularized Composite Allografts

\author{
Kadiyala V. Ravindra \\ Kadiyala.ravindra@duke.edu \\ Yash J. Avashia \\ Yash.avashia@duke.edu \\ Gina A. Mackert \\ Gina_alicia_mackert@yahoo.com \\ Brian May \\ Brian.may@dm.duke.edu \\ Detlev Erdmann \\ Detlev.erdmann@duke.edu
}

1 Division of Plastic, Reconstruction, Maxillofacial and Oral Surgery, Department of Surgery, Duke University Medical Center, DUMC 2824, Durham, NC 27710, USA

2 Duke University School of Medicine, Durham, NC 27710, USA

3 Division of Plastic, Reconstruction, Maxillofacial and Oral Surgery, Department of Surgery, Duke University Medical Center, DUMC Box 3181, Durham, NC 27710, USA

4 Division of Abdominal Transplantation, Department of Surgery, Duke University Medical Center, DUMC 3512, Durham, NC 27710, USA abdominal wall graft was uncommon and successfully treated with standard measures. A comparison of each institution's operative technique, maintenance immunosuppression regimen, and rejection management provide insight regarding the feasibility of AW-VCAs. AW-VCA has proven to serve as a viable option for patients undergoing multivisceral or intestinal transplantation with a donor and recipient size mismatch. Further experience with AW-VCA will aid in better defining its indications and help standardize technique and management.

Keywords Abdominal wall - Vascularized composite allotransplantation · Composite tissue allotransplantation

\section{Introduction}

The procedure of abdominal wall vascularized composite allotransplantation (AW-VCA) has been a surgical reality for over a decade. However, its application has been limited, and there is potential for further definition in its science, technique, and indication.

With the success of the first hand transplant [1], the new field of vascularized composite allotransplantation became a distinct entity. As the highest rung on the "reconstructive ladder," VCA has the potential to serve as an option for defects that have exhausted all other reconstructive options using biomaterial or autologous tissues. Over the past 15 years, more than 100 VCA transplants have been done worldwide. These include the hand [2], face [3], abdominal wall [4], laryngotracheal [5], tongue [6], knee and femur [7], and phallus [8] allografts. Most of the technical results of these surgical transplants have been successful [9]. While the literature has described several techniques for managing large abdominal wall defects, VCA thus far has been reserved for a select group of 
patients. At the time this manuscript is written, three institutions have reported a total of 21 AW-VCAs since 1999 in patients undergoing intestinal transplantation (IT) or multivisceral transplantation (MVT) $[4,10 \bullet, 11 \cdot 12,13$, 14.]. The application of the technique solely for abdominal wall restoration without a simultaneous solid organ transplant has not been reported, and the ethical issues that surround such clinical indication remain unaddressed.

\section{Background}

The foundation of organ transplantation was laid by Tom Gibson and Peter Medawar during World War II with the clinical goal of skin resurfacing and composite tissue reconstruction for the British pilots and seamen burned in U-boat attacks. Joseph Murray from Boston achieved the first clinical success in 1954 by transplanting a kidney between identical twins [15]. The field of allotransplantation expanded with improved knowledge of alloimmune response and advances in immunosuppression. By the turn of the century, clinical success in the liver, heart, lung, pancreas, kidney, and small bowel was widespread. It is only recently that VCA has been introduced as a potential clinical therapy.

AW-VCA has the largest skin component compared to other VCAs such as the hand and face [16]. A third of patients in the abdominal wall case series have presented with episodes of rejection [10•]. In the last two decades, research teams have performed various experimental studies that have led to a healthy record of VCAs of various types. These initial efforts have subsequently grown to create more than a dozen VCA programs worldwide.

\section{Anatomy}

The abdominal wall musculature works in a synkinetic fashion to protect abdominal viscera, increase abdominal pressure when required, and provide anterior support for the abdominal cavity.

The blood supply to the abdominal wall can be divided into three vascular zones that supply the overlying skin and subcutaneous tissues through musculo-cutaneous perforators. Zone I includes the upper and mid-central abdominal walls and is supplied by the superior epigastric artery (SEA) and deep inferior epigastric artery (DIEA) that lie within the rectus abdominis muscle. Inferiorly, the external iliac artery gives off two important vessels for abdominal wall vasculature, one of which is the DIEA. Zone II consists of the lower abdominal wall and is supplied by the epigastric arcade, which includes the superficial inferior epigastric artery (SIEA), superficial circumflex iliac artery (SCIA), and superficial external pudendal artery. Perforators from the deep circumflex iliac arteries
(DCIA) also supply the region of skin posterior and cephalad to the anterior superior iliac spine. The SIEA and SCIA arise from the femoral artery just below the inguinal ligament. The DCIA is the second of two vessels that arises from the external iliac artery, roughly $1 \mathrm{~cm}$ above the inguinal ligament. Zone III corresponds with the lateral abdominal wall musculature and is primarily supplied by the musculophrenic and lateral lumbar arteries.

Recent studies have commented on harvesting functional abdominal wall flaps to preserve its physiologic potential [17]. Motor innervation to the AW is supplied by the 6th through 12th intercostal nerves, iliohypogastric, and ilioinguinal nerves. Among the abdominal wall transplants performed, there have been two approaches to flap harvesting: microvascular and macrovascular $[4,11 \bullet]$. The first approach describes harvesting a cuff of the external iliac and/or femoral artery. The second approach describes harvesting the abdominal wall flap on the DIEA system. The latter approach implies a microvascular technique for anastomosis while the former requires a macrovascular technique (Fig. 1).

In the context of micro- and macrovascular approaches to AW-VCA, Hollenbeck et al. provided the first detailed anatomical study of abdominal wall vasculature [18・•]. The authors compared tissue perfusion of five fresh human cadaver abdominal wall flaps raised on either an external iliac and femoral artery cuff or the DIEA system. The authors described the value of the former since the cuff provides the origin of four sources of vascular inflow for the abdominal wall: DIEA, DCIA, SIEA, and SCIA. These vessels supply blood to Zones I and II as described above. In this study, the authors demonstrated a $34 \%$ increased perfusion of the abdominal wall with use of an iliac and femoral cuff. They reported poor superolateral wall perfusion, correlating with Zone III. Blood supply to this region is based on the musculophrenic and lumbar arteries, which would need to be harvested in order to reliably capture this vascular territory.

\section{Current Approaches to Large Abdominal Defects}

There are numerous causes for large abdominal wall defects (Table 1). The loss of abdominal domain is common in patients who have undergone massive or repeated intestinal resection, particularly when complicated by sepsis. The abdominal cavity is scarred and contracted, and the abdominal wall skin is often fibrotic from fistula, stomas, and prior operations.

The fascia and soft tissue envelope of the abdominal wall may be considered as two separate units. The musculofascial defect can be repaired by primary closure with or without component separation, fascial grafts, fascial components of tissue flaps, and synthetic or biologic mesh. Stable skin coverage can be provided with primary closure of the skin, skin 
Fig. 1 a This image shows the arterial supply to an AW-VCA harvested on the external iliac and femoral artery cuff giving rise to their respective highlighted branches (DIEA, DCIA, SCIA, and SIEA). b This image shows the arterial supply to an AW-VCA harvested on the highlighted DIEA system. Notice the regional correlation with Zone I and the relatively smaller area of perfusion compared to the AWVCA raised on an external iliac and femoral artery cuff a

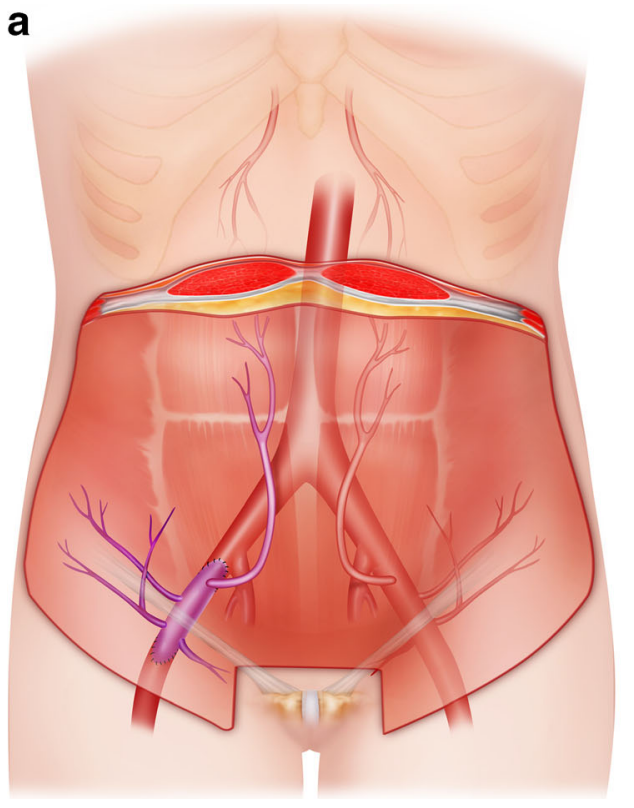

b

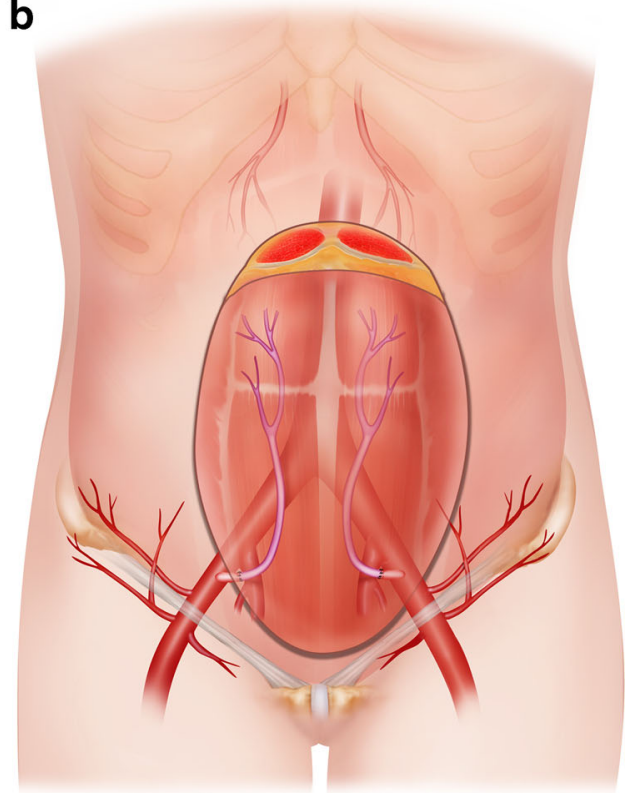

grafts, tissue expansion, local or regional flaps, or free tissue transfer.

For large fascial defects, component separation facilitates approximation of the musculofascial tissues in the midline [19]. The use of mesh in abdominal wall reconstruction has become the mainstay of repair. Options include synthetic mesh such as polytetrafluoroethylene (PTFE), polyglactin (Vicryl), or polypropylene (Prolene) [20]. These methods carry the risk of infection, adhesions, and enteric fistula [21].

Table 1 Causes of large abdominal wall defects

\begin{tabular}{ll}
\hline Tumor & Desmoid \\
& Dermatofibrosarcoma \\
Trauma & Soft tissue sarcoma \\
& Traumatic loss of abdominal wall \\
Abdominal wall necrosis & Necrotizing fasciitis \\
Vnfectious & Enterocutaneous fistula \\
Vascular & Vasculitis \\
Iatrogenic & Repetitive abdominal operations \\
& Repetitive abdominal wall reconstructions \\
& Multivisceral transplantation \\
Short gut syndrome & Intestinal transplantation \\
(candidate for intestinal & Necrotizing enterocolitis \\
transplant) & Crohn's disease \\
& Gastroschisis \\
& Midgut volvulus \\
& Omphalocele \\
& Jejunoileal atresia \\
& Internal hernia \\
& Congenital short bowel \\
& Meconium ileus \\
& Malrotation \\
& Intussusception \\
& Mesenteric ischemia \\
&
\end{tabular}

Biologic mesh is another alternative but has been associated with higher infection rates [22].

Another option has been the use of soft tissue flaps. Thighbased pedicle flaps include anterolateral thigh flap (ALT), tensor fascia lata flap, rectus femoris flap, vastus lateralis, gracilis muscle, or a combined thigh flap. Nearly the entire abdominal wall skin can be reconstructed with bilateral pedicle subtotal thigh flaps [23]. While these flaps have a fascial component, mesh is often preferred for reconstruction to avoid undue tension on the flap. For defects of the upper abdomen, pedicle flap options for soft tissue coverage are limited and include the latissimus dorsi muscle flap. Free flaps have been used for abdominal wall reconstruction in patients with extremely large defects in the central upper abdomen. Free flaps include tensor fascia lata, ALT, or other combined thigh flaps described above including the latissimus dorsi muscle. Tissue expansion is another viable option and can provide wellvascularized skin, subcutaneous tissue, and abdominal fascia. Risks include rupture, extrusion, infection, and expander failure. A review of intra-abdominal tissue expanders in 49 visceral transplants revealed a significant complication rate, which had prompted a shift to staged closure which is costly and time-consuming [24].

\section{Abdominal Wall Transplantation}

Varied techniques have been used for achieving coverage following bowel and multivisceral transplantation. Novel techniques using donor rectus fascia have been described. The use of rectus fascia as a free non-vascularized graft has been reported for transplant patients [25]. Wound infection was encountered in 7 out of 16 patients, with loss of graft in two 
patients. A modification of this technique has been the use of vascularized posterior rectus sheath in liver transplant patients. Agrawal et al. first reported the posterior rectus fascial allograft based off of the hepatic falciform artery (HFA) that runs in the falciform ligament [26]. The HFA arises as a terminal branch of either the middle or left hepatic artery.

These techniques are viable options, but they are inadequate in patients with massive tissue loss. They do not address the problem of skin coverage, which is also physiologically important. An important consideration in patients with massive abdominal wall loss is abdominal wall transplantation.

To date, all abdominal wall transplantations have been in conjunction with either an intestinal or multivisceral abdominal transplant. Abdominal wall transplantation is currently the second most common vascularized composite allograft performed with a total of $21 \mathrm{AW}-\mathrm{VCAs}$ reported worldwide [27]. Three institutions have published clinical experience in AW-VCAs: University of Miami, Bologna University (Italy), and Oxford University. There are a total of six publications that collectively report all three institutions' experience with abdominal wall transplants in a retrospective, case series fashion $[4,10 \bullet 11 \bullet, 12,13,14 \bullet]$.

Despite only three institutions having performed AWVCAs, their operative techniques are remarkably different. The University of Miami experience involved harvesting the abdominal wall in a heart-beating, brain-dead donor. The central part of the anterior abdominal wall including the rectus muscles with the rectus sheath and overlying skin was harvested. The vascular inflow was from the inferior epigastric vessels maintained in continuity with the iliac vessels [4]. Similar to a renal transplant technique, the donor iliac vessels were anastomosed to the recipient's common iliac artery and vein. In one patient, the graft was anastomosed to the infrarenal aorta and vena cava.

In contrast, Cipriani et al. (Bologna University) harvested the abdominal wall graft that included the rectus muscle along with both oblique muscles and parietal peritoneum. Vascularity was restored by anastomosing the donor DIEA to the recipients DIEA in an end-to-end fashion. They reported two aberrations to this technique. One donor flap had a single DIEA which proved to be sufficient for abdominal wall transplant. The second case presented with an inadequate DIEA in the donor, and the flap was anastomosed using the donor's DCIA also proving to be a viable option.

The technique described by the Oxford Group is innovative. In order to reduce cold ischemia time, the abdominal wall allograft was initially anastomosed to the recipient's nondominant forearm (ulnar artery) while the intestinal graft implantation was in progress [13]. At the completion of the intestinal transplant, the abdominal flap was transferred to the donor's DIEA or DCIA. Out of six patients, four were successfully transferred to the DIEA. The Oxford and Bologna techniques involve the use of the microvascular approach to
AW-VCA while the Miami experience reports the use of the macrovascular approach. The microvascular anastomotic technique preserves the donor iliac vessels, aorta, and vena cava for use by the solid organ transplant surgeons to facilitate liver, pancreas, and bowel transplantation. The outcomes from the three centers highlight certain differences and similarities (Table 2).

An important aspect of these three institutions' experience was their immunosuppression regimen, rejection rate, and overall flap survival. The Miami and Bologna centers used induction therapy with CAMPATH-1H (alemtuzumab) and maintenance immunosuppression with tacrolimus. Corticosteroids were not used in the maintenance regimen. A total of four doses of CAMPATH-1H were given: intra-operatively, immediate post-transplant, and on post-transplant days 3 and 7. The Oxford Group used similar induction with two doses of CAMPATH-1H (6-h post-reperfusion of the bowel and 2nd dose $24 \mathrm{~h}$ later) and maintenance immunosuppression of tacrolimus and prednisolone. Despite this difference, both the Miami and Oxford Groups reported a similar number of rejection episodes (three and four, respectively).

The Oxford Group provided the description of rejection episodes and reported the results of skin biopsies based on the Banff grading system [13]. Rejection was associated with a characteristic "perifollicular, maculopapular/papular rash," and biopsies were graded as Banff grade II or III. The rejection events were treated with a combination of basiliximab, a 3day pulse of methylprednisolone, addition of azathioprine, and the use of topical tacrolimus. Acute cellular rejection was treated with intravenous pulse corticosteroids in the Miami experience. Both groups reported resolution of rejection with the above strategy.

From the reports, abdominal wall transplants required similar immunosuppression than the one used as a standard of care for IT or MVT [13]. Rejection episodes and their management are similar to that of solid organs, and similar to other organs, VCA appears to have comparative survival rates when immunosuppression protocols are followed properly [28, 29]. The incidence of metabolic complications and opportunistic infections are similar to solid organ transplantation [27, 29]. The intestine and the skin of the abdominal wall graft may reject independently. Therefore, monitoring the skin on the abdominal wall graft should not be utilized as a measure of reactivity toward the intestinal graft. Grafts are monitored for rejection using the standardized scoring system for vascularized composite allografts [30].

Overall, 2 of the 21 abdominal allografts failed postoperatively on days 1 and 6 secondary to vascular thrombosis. Neither of these episodes occurred in patients with microvascular anastomoses. There were further reports of graft loss during the post-operative period. The overall patient mortality rate was 56,33 , and $17 \%$ for the three groups. The reported causes of mortality included sepsis, intestinal transplant 
Table 2 Outcomes of abdominal wall vascularized composite tissue allotransplantation

\begin{tabular}{|c|c|c|c|}
\hline & University of Miami $[4,10 \bullet, 12]$ & Bologna University $[11 \bullet, 12]$ & Oxford University $[13,14 \bullet]$ \\
\hline Patients $(n)$ & 11 & 3 & 6 \\
\hline Gender (M:F) & $6: 5$ & $2: 1$ & $4: 2$ \\
\hline Age range & $1-53$ & $33-41$ & $27-72$ \\
\hline Pediatric (age < 15) & 4 & 0 & 0 \\
\hline AW-VCA (no.) & 12 & 3 & 6 \\
\hline IT:MVT (no.) & $6: 5$ & $3: 0$ & $6: 0$ \\
\hline Technique (DIEA:common iliac) & $0: 12$ & $3: 0$ & $6: 0^{\mathrm{b}}$ \\
\hline Flap size $\left(\mathrm{cm}^{2}\right)$ & $150-500$ & 350 & NR \\
\hline Warm ischemia (min) & NR & NR & 50 and 61 \\
\hline Cold ischemia (min) & NR & NR & 305 \\
\hline Op time (min) & Added 120 & $105-150$ & NR \\
\hline Follow-up (month) & $2-84$ & 12 & 12 \\
\hline Immunosuppression & $\begin{array}{l}\text { Alemtuzumab }^{\mathrm{a}} \\
\text { Tacrolimus } \\
\text { No corticosteroids }\end{array}$ & $\begin{array}{l}\text { Alemtuzumab } \\
\text { Tacrolimus } \\
\text { No corticosteroids }\end{array}$ & $\begin{array}{l}\text { Alemtuzumab } \\
\text { Tacrolimus } \\
\text { Corticosteroids }\end{array}$ \\
\hline Rejection regimen & IV corticosteroid & IV corticosteroid & $\begin{array}{l}\text { Basiliximab } \\
\text { Pulsed IV corticosteroids over } 3 \text { days } \\
\text { Topical tacrolimus ointment } \\
\text { Addition of azathioprine to regimen }\end{array}$ \\
\hline Report rejection episodes & $4(36 \%)$ & 0 & $3(50 \%)$ \\
\hline Resolution of rejection episode & $4(100 \%)$ & 0 & $3(100 \%)$ \\
\hline Flap survival (\%) & 83 & 100 & 100 \\
\hline Overall mortality (\%) & 56 & 33 & 17 \\
\hline
\end{tabular}

$N R$ not reported

${ }^{a}$ One patient received daclizumab followed by tacrolimus and prednisone

${ }^{\mathrm{b}}$ Abdominal flap harvested on DIEA system and was transferred to ulnar artery while intestinal graft was being placed. AW-VCA was then transferred to either DIEA or DCIA system. For the two cases in which the flaps remained on ulnar artery, one was later transferred to LIMA via vein loop

rejection, primary non-function of intestinal transplant, and lymphoproliferative disease.

Interestingly, muscular hypotrophy and the absence of muscular tone in AW-VCAs were attributed to severed nerves. None of the flaps were harvested with nerve grafts. Authors concluded that reconstruction of the abdominal wall graft innervation is not necessary because even though patients had developed hypotrophy of the muscular layer of the graft, there was no significant hernia formation.

\section{Current Research}

Experimental studies specific to AW-VCA have focused on testing VCA antigenicity, surgical techniques, and immunosuppression regimens in reproducible animal models. While animal studies do serve as a viable option, direct clinical practice serves as the gold standard to assessing outcomes and nuances with immunosuppression. Jin et al. demonstrated the bio-mechanic and histologic changes that occur in AWVCA using a porcine model [17]. The authors reported that the abdominal graft developed significant muscular atrophy, decreased tensile strength, and increased stiffness after 28 days. While the skin looked normal on gross examination, there was histological evidence of rejection that correlated with the clinical reports on humans. While there are no reports of hernia in AW-VCA patients, the authors concluded that these functional changes seen in the transplanted abdomens would lead to increased diastasis and hernia formation over time.

Frequent VCA rejection has been attributed to an increased antigenicity of skin. Nasir et al. was the first to experimentally study this notion by introducing a rat model for abdominal wall transplantation and monitoring immunologic responses to high skin antigenic loads [16]. After directly comparing various allograft flap surface areas in this model, the authors reported that the total abdominal wall graft has the largest area of skin compared to other VCA grafts including the total face, limb, hemiface, and groin. They suggest that the level of donor hematopoietic cells that pass from allograft to the recipient may be in proportion to the flap dimensions. By using flow cytometry, they assessed the presence of donor-specific chimerism for donor $\mathrm{MHC}$ class I $\left(\mathrm{RT}^{\mathrm{n}}\right)$ antigen in the 
peripheral blood of the abdominal wall transplant recipients. Chimerism declined from post-transplant day $7(6.7 \%)$ to post-transplant day $100(1.31 \%)$. Chimerism levels from highest to lowest were the hemiface, total abdominal wall, and groin flap. The highest level seen in face was attributed to a higher amount of lymphatic tissue associated with the allograft.

To expand upon the previous report on donor-specific chimerism, Quigley et al. performed studies using a rat model for full thickness total abdominal wall transplants based on a single pedicle between fully MHCmismatched rat strains [31]. Post-transplant flow cytometry did not reveal donor-specific chimerism in any of the groups, which was attributed to the lack of bone marrow or lymph node components in the transplanted tissue. The authors had additionally tested three different dosages of tacrolimus for immunosuppression. These were administered intraperitoneal on the transplant day until post-transplant day 100. After developing a visual assessment scale for rejection, authors found that the groups with higher dosages did not show signs of rejection while the lower group displayed mixed responses. Histological examination correlated with these findings. They furthermore demonstrated the viability of an AW-VCA on a single pedicle, which was also demonstrated by the Italy Group in one patient [11•].

In order to investigate tolerance further, Ramirez et al. used a rat animal model and performed VCAs of a hemi-abdominal wall along with a hind limb osteomyocutaneous flap (HLOMC) [32]. They found that an isolated HLOMC flap had a prolonged course to rejection compared to isolated abdominal wall or combined AW-VCA and HLOMC. This suggests that the beneficial effects of vascularized bone marrow transplant on VCA survival may be limited by a certain mass of allograft that it can support.

\section{Ethics Associated with AW-VCA}

Face transplantation has undergone an intense ethics debate since the first reported case in 2005 [3]. Even though AW-VCA is the second most performed VCA today, it has not been included in these discussions likely due to the exit strategies available in the case of graft loss. In a systematic review by Kiwanuka et al., the authors showed a time-related trend of "in favor" publications increasing from 0 to $100 \%$ by 2008 in regards to face transplantation [33]. After evaluating all publications on this topic, they developed a set of 15 core topics pertaining to the ethical debate. These include (1) identity, (2) immunosuppression, (3) risks/benefits, (4) surgery/life-improving, (5) society's role, (6) failure of transplant, (7) quality of life, (8) donation, donor's family, (9) informed consent, (10) importance of face, (11) patient selection, (12) ethical principles, (13) future use, (14) cost, and (15) disfigured people cannot live life regularly.

Of the above mentioned issues, only a select few are applicable to AW-VCA. Inherent to this procedure, the concern for identity is not as important due to the anatomic location with the abdomen being frequently covered by clothing. Furthermore, the pre-operative abdomen is often times in a worse predicament than the post-transplant abdomen. In fact, one may argue a significant improvement in quality of life from a functional and cosmetic perspective for these patients. The risk vs. benefit ratio between an AW-VCA and immunosuppression is more balanced when the AW-VCA is combined with an intestine or multivisceral transplant. However, this risk will be relevant to the debate when abdominal wall transplantation is done in isolation. Expanding use of AW-VCA solely for abdominal wall defects without concomitant solid organ transplantation may require further developments in immunosuppression and tolerance protocols. With no reported flap failure or technical complications with AW-VCA along with mortality rate which may be predominantly attributed to the patient's intestinal or multivisceral transplantation, it is worthwhile raising the question, "Which patient may be a candidate for AW-VCA?"

\section{Conclusion}

AW-VCAs have shown to be a viable option thus far for patients undergoing intestinal or multivisceral transplantation. Prior clinical experience coupled with cadaveric studies and animal studies have better elucidated nuances regarding the anatomy, technique, and immunology. Inherent to this topic are the potential indications for AW-VCA given the various etiologies for large abdominal wall defects. Further experience and research on AW-VCA will aid in better defining its indications and help standardize technique and management.

Acknowledgments The authors would like to acknowledge Stan Coffman for the illustration in this article.

\section{Compliance with Ethics Guidelines}

Conflict of Interest YJ Avashia, GA Mackert, B May, Cendales LC, D Erdmann, and KV Ravindra declare that they have no conflict of interest.

Human and Animal Rights and Informed Consent This article does not contain any studies with human or animal subjects performed by any of the authors. 


\section{References}

Papers of particular interest, published recently, have been highlighted as:

- Of importance

•- Of major importance

1. Dubernard JM, Owen E, Herzberg G, et al. Human hand allograft: report on first 6 months. Lancet. 1999;353(9161):1315-20.

2. Dubernard JM, Long H, George JL, et al. Study of the function of a renal transplant during removal from the donor (author's transl). J Urol Nephrol. 1974;80(10-11):814-23.

3. Dubernard JM, Lengele B, Morelon E, et al. Outcomes 18 months after the first human partial face transplantation. N Engl J Med. 2007;357(24):2451-60.

4. Levi DM, Tzakis AG, Kato T, et al. Transplantation of the abdominal wall. Lancet. 2003;361(9376):2173-6.

5. Strome M, Stein J, Esclamado R, et al. Laryngeal transplantation and 40-month follow-up. N Engl J Med. 2001;344(22):1676-9.

6. Birchall M. Tongue transplantation. Lancet. 2004;363(9422):1663.

7. Hofmann GO, Kirschner MH, Wagner FD, Land W, Buhren V. Allogeneic vascularized grafting of a human knee joint with postoperative immunosuppression. Arch Orthop Trauma Surg. 1997;116(3):125-8.

8. $\mathrm{Hu} \mathrm{W}, \mathrm{Lu}$ J, Zhang L, et al. A preliminary report of penile transplantation. Eur Urol. 2006;50(4):851-3.

9. Tobin GR, Breidenbach 3rd WC, Pidwell DJ, Ildstad ST, Ravindra KV. Transplantation of the hand, face, and composite structures: evolution and current status. Clin Plast Surg. 2007;34(2):271-8. ix-x.

10. Selvaggi G, Levi DM, Kato T, et al. Expanded use of transplantation techniques: abdominal wall transplantation and intestinal autotransplantation. Transplant Proc. 2004;36(5):1561-3. This study presents experience with abdominal wall transplantation with both intestinal and multivisceral transplantation. Details regarding immunosuppression regimen are discussed. The complication of venous outflow thrombosis is included in this article.

11. Cipriani R, Contedini F, Santoli M, et al. Abdominal wall transplantation with microsurgical technique. Am J Transplant Off J Am Soc Transplant Am Soc Transplant Surg. 2007;7(5):1304-7. This article portrays the feasibility of microsurgical abdominal wall transplantation without the need to procure larger vessels including iliac vessels, aorta, and vena cava.

12. Selvaggi G, Levi DM, Cipriani R, Sgarzani R, Pinna AD, Tzakis AG. Abdominal wall transplantation: surgical and immunologic aspects. Transplant Proc. 2009;41(2):521-2.

13. Allin BS, Ceresa CD, Issa F, et al. A single center experience of abdominal wall graft rejection after combined intestinal and abdominal wall transplantation. Am J Transplant Off J Am Soc Transplant Am Soc Transplant Surg. 2013;13(8):2211-5.

14. Giele H, Bendon C, Reddy S, et al. Remote revascularization of abdominal wall transplants using the forearm. Am J Transplant Off J Am Soc Transplant Am Soc Transplant Surg. 2014;14(6):1410-6. This is a case series of six patients that underwent intestinal and abdominal wall transplantation. The technique presented is unique to the other techniques described for abdominal wall transplantation. This article also provides results of punch biopsies during post-operative rejection episodes.

15. Murray JE. Organ transplantation (skin, kidney, heart) and the plastic surgeon. Plast Reconstr Surg. 1971;47(5):425-31.
16. Nasir S, Bozkurt M, Klimczak A, Siemionow M. Large antigenic skin load in total abdominal wall transplants permits chimerism induction. Ann Plast Surg. 2008;61(5):572-9.

17. Jin J, Williams CP, Soltanian H, et al. Use of abdominal wall allotransplantation as an alternative for the management of end stage abdominal wall failure in a porcine model. J Surg Res. 2010;162(2): 314-20.

18.• Hollenbeck ST, Senghaas A, Turley R, et al. The extended abdominal wall flap for transplantation. Transplant Proc. 2011;43(5): 1701-5. This article presents a unique and valuable anatomical study for abdominal wall transplantation. This cadaveric study seeks to determine the maximal abdominal skin surface available through either an external iliac/femoral cuff-based pedicle versus a deep inferior epigastric artery pedicle.

19. Ramirez OM, Ruas E, Dellon AL. Components separation method for closure of abdominal-wall defects: an anatomic and clinical study. Plast Reconstr Surg. 1990;86(3):519-26.

20. Ravindra KV, Martin AE, Vikraman DS, et al. Use of vascularized posterior rectus sheath allograft in pediatric multivisceral transplantation-report of two cases. Am J Transplant Off J Am Soc Transplant Am Soc Transplant Surg. 2012;12(8):2242-6.

21. An G, Walter RJ, Nagy K. Closure of abdominal wall defects using acellular dermal matrix. J Trauma. 2004;56(6):1266-75.

22. Allison AC, Eugui EM. Immunosuppressive and other effects of mycophenolic acid and an ester prodrug, mycophenolate mofetil. Immunol Rev Dec. 1993;136:5-28.

23. Lin SJ, Butler CE. Subtotal thigh flap and bioprosthetic mesh reconstruction for large, composite abdominal wall defects. Plast Reconstr Surg. 2010;125(4):1146-56.

24. Gupte GL, Haghighi KS, Sharif K, et al. Surgical complications after intestinal transplantation in infants and children-UK experience. J Pediatr Surg. 2010;45(7):1473-8.

25. Gondolesi G, Selvaggi G, Tzakis A, et al. Use of the abdominal rectus fascia as a nonvascularized allograft for abdominal wall closure after liver, intestinal, and multivisceral transplantation. Transplantation. 2009;87(12):1884-8.

26. Agarwal S, Dorafshar AH, Harland RC, Millis JM, Gottlieb LJ. Liver and vascularized posterior rectus sheath fascia composite tissue allotransplantation. Am J Transplant. 2010;10(12):2712-6.

27. Petruzzo P, Dubernard JM. The International Registry on Hand and Composite Tissue allotransplantation. Clin Transpl. 2011;247-253.

28. Breidenbach 3rd WC, Tobin 2nd GR, Gorantla VS, Gonzalez RN, Granger DK. A position statement in support of hand transplantation. J Hand Surg [Am]. 2002;27(5):760-70.

29. Thaunat O, Badet L, El-Jaafari A, Kanitakis J, Dubernard JM, Morelon E. Composite tissue allograft extends a helping hand to transplant immunologists. Am J Transplant. 2006;6(10):2238-42.

30. Cendales LC, Kirk AD, Moresi JM, Ruiz P, Kleiner DE. Composite tissue allotransplantation: classification of clinical acute skin rejection. Transplantation. 2006;81(3):418-22.

31. Quigley MA, Fletcher DR, Zhang W, Nguyen VT. Development of a reliable model of total abdominal wall transplantation. Plast Reconstr Surg. 2013;132(4):988-94.

32. Ramirez AE, Cheng HY, Lao WW, et al. A novel rat full-thickness hemi-abdominal wall/hindlimb osteomyocutaneous combined flap: influence of allograft mass and vascularized bone marrow content on vascularized composite allograft survival. Transpl Int. 2014;27(9):977-86.

33. Kiwanuka H, Bueno EM, Diaz-Siso JR, Sisk GC, Lehmann LS, Pomahac B. Evolution of ethical debate on face transplantation. Plast Reconstr Surg. 2013;132(6):1558-68. 